\title{
Towards a Home-based Virtual Reality Game System to Promote Exercise
}

\author{
Ashish Amresh ${ }^{1} \&$ Rahul Salla ${ }^{2}$ \\ ${ }^{1}$ School of Computing, Informatics and Decision Systems Engineering, \\ Arizona State University \\ amresh@asu.edu \\ ${ }^{2}$ The Decision Theater, \\ Arizona State University \\ rahul.salla@asu.edu
}

\begin{abstract}
This paper presents the design and usability testing results for a home-based virtual reality game system that aims to promote exercise with the spin machine. The novelty of our approach lies in the design of the game mechanics that promote increased calorific output among the participants and the ease of setup with any commercially available Virtual Reality (VR) sensor. The paper describes the motivation behind the developed system, the design details of the games created to promote the use of the system and the results from a usability test conducted with 37 participants.
\end{abstract}

\section{Introduction}

While the purpose of the gym is to provide the user with a facility that can provide a means to perform various exercises that aim to stimulate several body functions under one roof, it severely lacks the ability to transform the experience to mimic reality. Real-world exercises on the other hand provide a multitude of options for working out as opposed to the gym wherein one location cannot possibly provide the experience and the depth that is achieved by working out in the real world. A traditional exercise in the real-world comes with an experience that is usually unique; whereas in the gym it is a constant experience amongst all facilities. An example of this static experience is your typical "spin-machine", where you sit on a stationary bike and ride with a desired speed and resistance. The experience of this is mostly staring straight forward with no visual/mental stimulation. Many gyms have opted to install TV screens and music-players to enhance this experience, however adding a visual medium to augment the static experience does not solve the problem and we postulate that a "fully" immersive simulation that includes an augmented/virtual reality based experience along with exercise will produce desirable outcomes when it comes to participation, engagement and enjoyment.

By introducing the aspects of a video game into fitness we aim to encourage users to stay fit and workout more often. Through competition (various obstacles such as monsters chasing the user around), and being able to do the physically impossible (being able to float in space, ride the bike upside down, etc.), the user will be more inclined to work out because the virtual reality system will make it more entertaining. By creating a selection of levels to interactively explore using virtual reality (VR), we can measure their enjoyment and change in exercise behaviors using surveys before and after exercising with and without VR. In addition, we can record the calories-burned using the built-in instrument for measuring caloriesburned in the exercise bikes that we use. By comparing this data together and receiving feedback personally from our subjects we can generate an idea of just how much VR impacts their workout routine. In this paper we provide the results of the design of a VR based gaming system that has been built to be able to run in a home-based environment with minimal set-up time and cost. We start by providing the background information in this up and coming area of "exergaming". We then look at current practices and examples in this area and the barriers facing the wide adoption of such set-ups in homes. We discuss the unique aspects of our game design methodology, our experimental set-up, the results from our test and conclude by providing future direction for this area.

\subsection{Background and theoretical basis}

There has been a great push in the last decade to develop game based applications that are for nonentertainment purposes. This has led to the creation of the field of "serious games" [1]. While serious gaming can encompass many fields ranging from education, training, military and healthcare, there has been further development in game based techniques that has led to the creation of terms such as game based learning (GBL) [2], gamification [3] and exergaming [4]. While there are overlapping similarities between these terms and have been used synonymously, there are very stark differences that need to be noted. For instance, there is no direct learning provided by gamification and exergaming applications and many games that are 
designed to educate often do not include gamification elements. In the context of this paper, it is important to note that exergaming can be closely linked or even termed as a gamification method, as it is aimed at improving a particular outcome by providing to the end-user a rewards based platform. Gamification in healthcare has gained popularity both in research as well as industrial and clinical applications [5, 6, 7]. Exergaming on the other hand has seen limited adoption in clinical settings. In [8] a study was conducted that showed that exergaming could increase physical activity levels in children and in [9] a similar study with college students showed positive results. [10] provides a complete list of exergaming contexts and their use in a variety of settings and environments. It has been observed that VR based exergaming setups are not very common and almost non-existent in the home environment. The primary theoretical basis behind the design and implementation of exergaming applications is the theory of flow [11] and intrinsic motivation [12]. This has been proven to work by using exergaming in obese adolescents [13], elementary school children [14] and older adults [15]. According to this theory, an exergaming application when designed with the principles of flow and intrinsic motivation develops acceptance, repetition, persistence and skill-development in the user and encourages sustained and long-term participation. In this paper we present specific game design mechanics via three distinct game levels that incorporate the theory of flow and intrinsic motivation and make the game fun to play, competitive, challenging and rewarding.

\subsection{Virtual reality alternatives}

There have been several exercise systems that have been developed as alternatives to VR. The aim of these systems is to improve engagement and make the process of using an exercise bike or a spin machine more enjoyable. The Expresso [16], similar to our system, is meant to enhance the user's workout by presenting a virtual world the user can bike in. The main difference is that our system utilizes VR with an Oculus Rift [17], whereas their product is a bike with a screen attached to it. This means that users of the Expresso do not get the full immersive experience of the Oculus Rift. However, because the Expresso does not use a VR headset, users will have more situational awareness of their surroundings. The VirZoom [18] uses both the bike and integrated VR headset, and it uses the bike to move the in-game character forward or backward. There are buttons on the handles of the bike which players can use to steer and perform other in game interactions. The main difference between our system and the VirZoom, is that the VirZoom is designed to enhance gaming, rather than focus on physical exercise. In VirZoom users control movement using the bike, and can indeed get a workout from the bike, however the main purpose is to augment gaming, and not necessarily meant for serious exercise.

\section{Game Design}

Before developing the game, we sent out a survey to various potential users who might be interested in the idea of "gamifying" a workout. The purpose of the survey was to gather general information regarding how people would feel about "gamifying" physical exercise and whether using an Oculus Rift would entice users to go to a gym for a workout more frequently. There were a total of 41 participants, 21 males and 20 females. The survey recruitment took place at a fitness facility and most participants have a high affinity towards exercise with majority of the participants working out form more than 6 hours per week. $63 \%$ of the participants indicated that their primary form of workout was cardio and indicates that the survey results are reliable and the target audience is appropriate. $84 \%$ were familiar with VR systems and $62 \%$ indicated that they would use a VR system to augment their exercise equipment. This gave us a strong sense of purpose to build our system and carry out further development.

The games were designed and built using Unreal Engine 4 [19], a game engine for designing highfidelity video-games and provides the option to export the games into a VR device. We designed three custom levels that accentuate different levels of realism, exercise-type, and visual stimulation. From the initial user survey, cardio based exercise was the most commonly used as a form of workout so we choose the spin machine as our exercise system to augment VR with. The treadmill was the more popular form of cardio equipment used by the survey participants, but we chose the spin machine because it requires the user to be stationary to reduce the risk behind having the user run without a perception of where their feet are. After choosing the spin machine as our equipment, we began to design several game levels with aim of reaching a larger audience. We created a level for simulating how you can defy reality on a bike (Forest), a level for those that like action-oriented situations (Office), and a realistic level for non-gamers (Snow Mountain). In each level created, we developed AI to either chase the user or interact with the user in order to help "gamify" the workout and keep the user entertained.

The first level that is intended to be played by the user is a tranquil forest level with rolling hills. This 
level is built with a track that loops around as a circuit. The purpose of this level is to test how long the user is able to ride the bike in an environment that repeats itself. This is to help test how long the user will be interested in the simulation when the environment repeats itself and loops. During the ride of this track, various obstacles, such as AI that are trying to slow the character down by either colliding with the player or hitting the player. This track also has several vertical loops where users will be given the perspective of being completely upside down. We have included this to see how users react to movements that could be disorienting such as being upside down or banking quickly around a corner. The overall design of the track is a large, open map where the user can see their surroundings easily while riding the spin machine. The level, due to the change in pace is intended to be an aerobic exercise. Aerobic exercise is a type of workout focused on the cardiac muscles. This stimulates the user's heart and breathing rate in order to strengthen them. The goal is for the user to continue for a longer period of time at a consistent pace. Figure 1 shows the screenshots for the forest level.

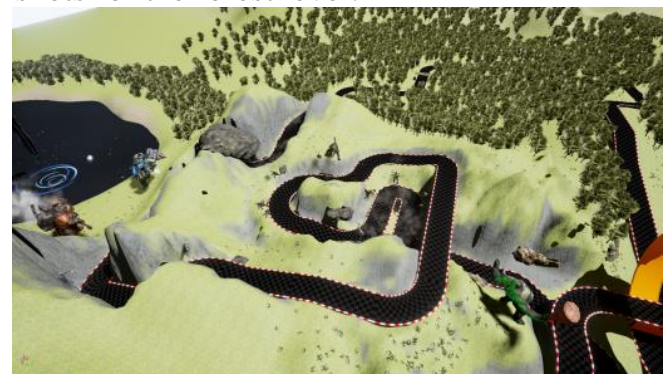

Figure 1. The forest level

In the second level the user is situated in an office building. The general layout of this level is a confined space with action scenes that manifest as the player progresses in the level. This level is designed to help test how users react to interactions in a closed space with a several pieces of action/visual clutter occurring in the background. This level is not intended to be played any longer than two minutes because its objective is a high intensity and short duration workout. This style of workout is to burn as many calories in a short amount of time.

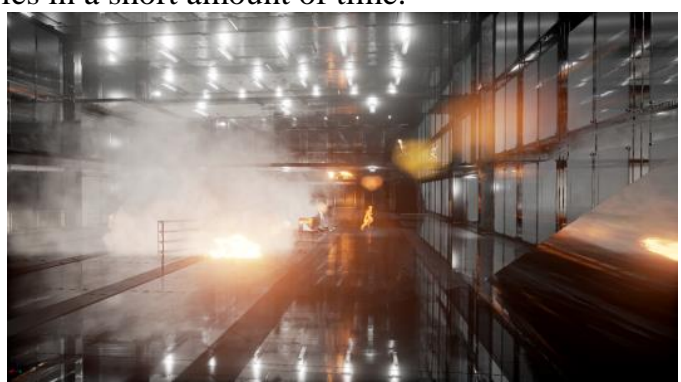

Figure 2. The office level
To help encourage the user to work out in this level, it is laid out with multiple AI encounters to immerse the user as if they are trying to escape the office building within a certain amount of time. The building is also on fire adding to the urgency to complete the level in a certain amount of time. Figure 2 shows the screenshot for the office level.

The final level in our game is a large, open, forested snow mountain. The general layout of the level is to help test the user on how they react to a level that is open with steep cliffs and dense forests. The level is the largest of the three levels. It is intended to be a long duration workout and the user is free to choose their pace. The pace and calorific output are designed to vary continuously based on several situations that include wild beasts that will chase the user for a certain duration of time to promote the change in pace. Figure 3 shows the screenshot for the snow mountain level.

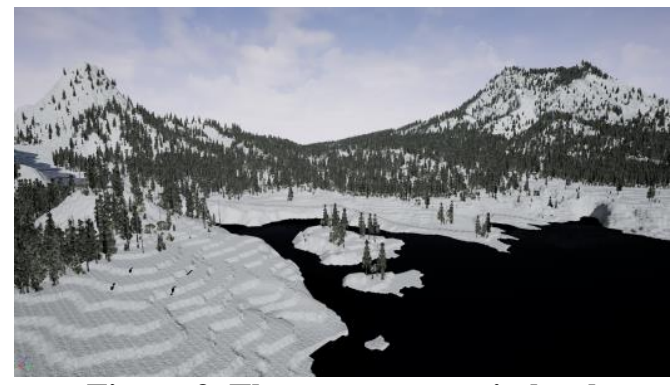

Figure 3. The snow mountain level

\section{Experimental Set-up}

The first challenge was to determine the appropriate method for player motion. There wasn't any reliable literature in this area and some studies [20, 21] had suggested that tracking head motion can correlate to the speed at which a person would pedal a bike or a spin machine. A prototype was set up to measure head movement and after an initial user study with a few participants it became evident that this was not reliable for the following reasons: 1) averaging head movement was not an accurate measure for pedal speed. 2) the built in VR sensor is not reliable to translate lateral vs other types of head movements and there was noise that had to be removed in the tracking data before making it reliable. 3) differences in participant's gender, height and age weighed in to the correlation methods.

We overcame the challenge by integrating a Arduino chip with an infrared sensor to the bike [23]. Every refresh of the game frame (typically 30 times a second), the Arduino sends a binary value (1: tripped; 0 : not-tripped) via the serial port to the gaming system. 
A tripped value indicates that the pedal has passed the infrared sensor. This gave us a time measure between two tripped values inside out game setting and help determine the speed of the motion. Figures 4 and 5 show the setup of the Arduino along with the spin machine and the Oculus VR sensor.

This prototype was tested on a small group of participants and the motion algorithm was accurate and reliable under several testing conditions and the demographic information of the user didn't influence the system. Initial observations reveled that there was initial discomfort in the users due to the sudden twists and turns in the forest level and in order to ease the user into getting familiar with the VR environment, the speed was damped around to the turns and circuits to avoid sudden movement. This damping factor was slowly removed in subsequent levels.

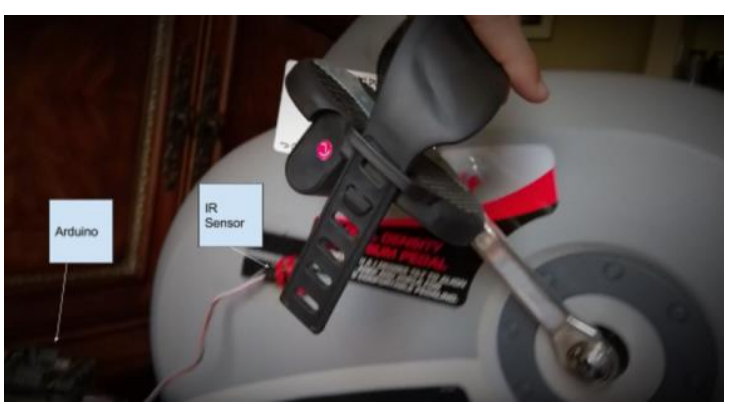

Figure 4. Arduino setup

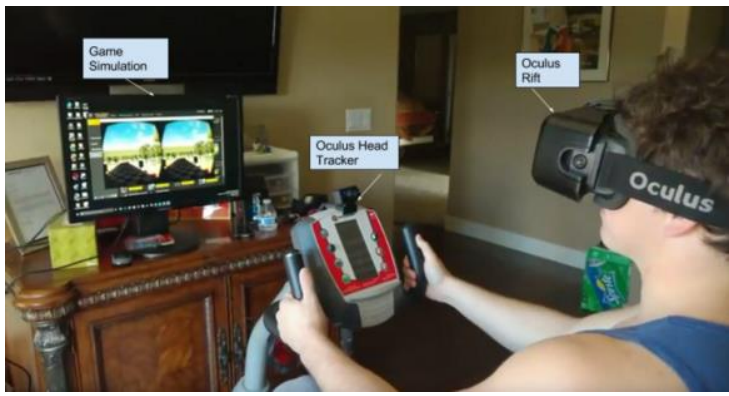

Figure 5. VR setup with Oculus

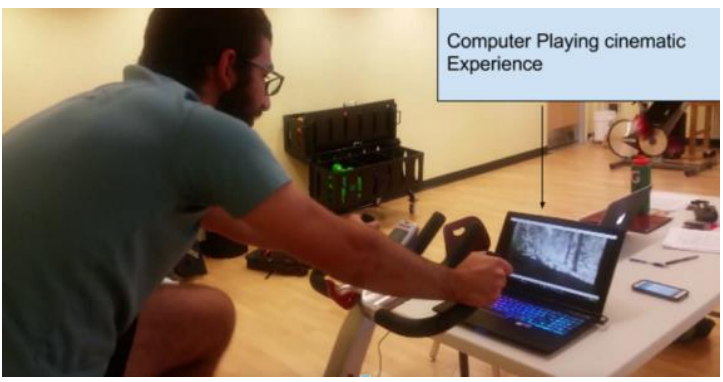

Figure 6. Cinematic experience

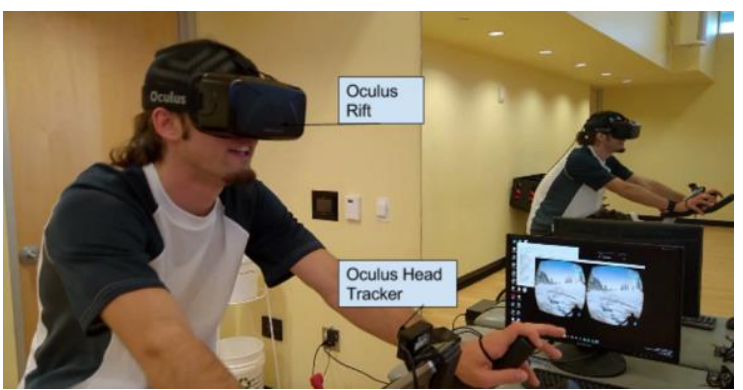

Figure 7. VR experience

The final usability was conducted inside a gym where three different testing stations were setup to conduct these tests. Station-one was a test to see how the user works out without any form of media (music, television, etc.). Station two (Figure 6) was a cinematic experience. This was to test how the user would perform on their workout using a television screen that is having them ride a bike on a trail through a video (completely non-interactive). Station-three (Figure 7) was the spin machine set up to work with the Oculus Rift. With all three stations, users would ride the spin machines for a total of 6.5 minutes, and then we recorded their calories burned during the exercise. We then conducted a survey to measure the user experience. The users played all three levels and the major objective of this user test was to perform an unbiased review of our system for both usability, reuse and ability to promote or increase calorific output.

\section{Results and observations}

A total of 37 participants were recruited (22: nonVR and 15: VR) for the study. One of the common requests for the game was to increase the amount of content provided in the game, people enjoyed playing it and wanted more in their experience or in other words 6.5 minutes was not enough for majority of the participants. Another observation is that some individuals were disoriented for the first minute while wearing the VR headset. One potential cause of that is the Oculus Rift itself. We were using the Developer Version DK2 [22] which has a lower resolution screen. As a result, the game, while being ran, will appear blurry. Another potential variable that may have caused bias of our results were the physical condition of the users. Some were just finishing up their workout at the gym and came to test after. Due to this, they may have burned more calories if they were not already fatigued from a previous workout. The users who were not disoriented were individuals identifying themselves as gamers; while the users who were disoriented were not self-proclaimed gamers. The users that were disoriented noted that they felt dizzy due to not being physically connected to the game, so when they 
moved, it threw them off. An example of this is that when turning around a bend in the track, a bicyclist to have to "bank" into the turn, whereas the users simply pedal forward with no feedback from in-game turning. Another observation that was noted was based on the intentional level designs. We had three different levels to test the user in various ways. The users who classified themselves as gamers tended to enjoy the Office Level due to the action sequences, while the non-gamers did not enjoy it but rather liked the more open levels. Part of the reason was due to the map being more open and spacious, making them feel less disoriented because there were less sharp turns. From this observation we can conclude that providing a wide variety of level types is ideal in order to make sure that a wide variety of user-interest is met.

When comparing the amount of calories burned between with VR and without VR (Figure 8), it is immediately noticeable that the average user with the Oculus Rift (Orange Line) is higher than the average user without the Oculus Rift (Gray Line). This supports our hypothesis that on average people will burn more calories with the Oculus rather than without. The data also shows that for the upper-end outliers, people without the Oculus Rift burned on average more calories, however, the standard deviation was higher for Oculus Rift users ( $75 \mathrm{KCals})$ compared to the non-Oculus Rift users ( $\sim 68 \mathrm{KCals})$; even though there were two upper-end outliers for non-Oculus Rift users compared to the ones with the Oculus Rift. It is also notable that on the lower-end of the spectrum, nonOculus Rift users burned on average more calories than Oculus Rift users. This would indicate that on average, most users burned more calories with the Oculus than without, however users who workout harder than the average and not as hard as the average will burn less calories on average, which would indicate the oculus distracts them from the workout therefore lowering performance.

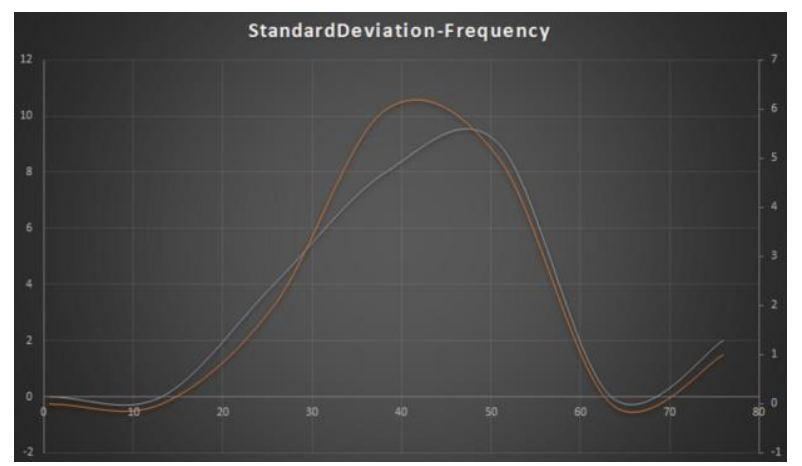

Figure 8. Calorific output comparison between VR and non-VR setups
The system has issues that can be solved with time. The price of the hardware required to run the Oculus is expensive. A computer capable of running it requires at a minimum a desktop with Nvidia GTX 970 video card or a laptop with Nvidia GTX 980M video card. The desktop is a cheaper solution but is less portable than a laptop. It would also benefit to have more sophisticated and accurate equipment for measuring users' caloric burn during the tests, as our method is approximation at best and does not account for particular builds of the persons involved. Additionally, if we had a larger userbase to perform tests on it would be more appropriate to test users exclusively exercising with the cinematic experience, VR experience, and a standard workout with no media, to eliminate all bias that could influence them; such as the user comparing non-VR versus VR directly and padding their opinion in response. In future iterations it would also be nice to have a more embedded and accurate form of movement measurement via a gear installed on the bike-pedal. This would allow the translation of movement from the real-world to in-game nearly 1-to1 rather than approximating the speed and revolutions per minute of the pedal.

Usability results indicated that $75 \%$ of VR users will use the system along with their workouts and $65 \%$ of these users indicated that they would use it for longer periods of time. Figures 9-15 provide detailed results from the usability test.

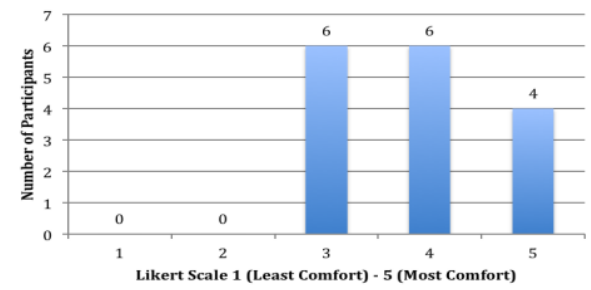

Figure 9. How comfortatble was the VR

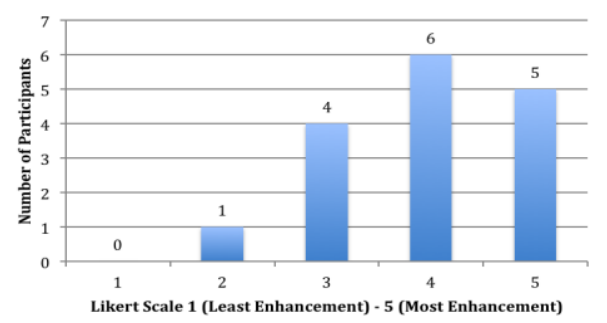

Figure 10. Did VR enhance your workout experience 


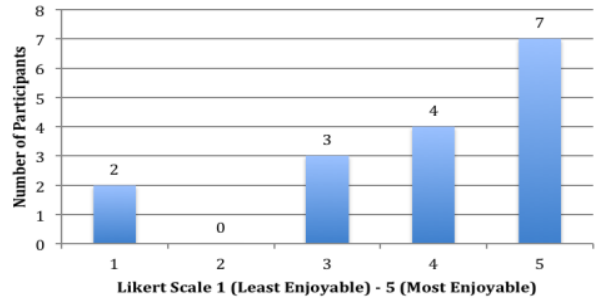

Figure 11. Was your workout more enjoyable with VR

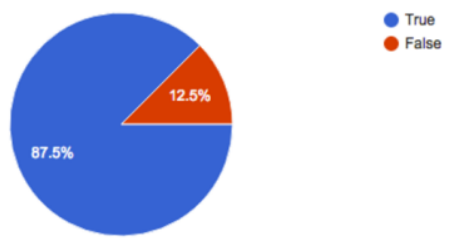

Figure 12. Would you exercise more if there were many unique levels

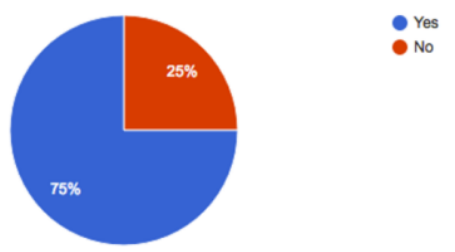

Figure 13. Would you use VR again

\section{Conclusions}

Based on our results provided through all the testing sessions we held, implementing virtual reality to "gamify" fitness machines helps enhance the overall workout. Of the users tested, $75 \%$ were interested in using the Oculus Rift again to workout because they enjoyed it and was fun to play and repeat. From the results of the test, the user's workout was not hindered due to the Oculus rift and some of the users who identified themselves, as gamers were competitive in beating others that were being tested. One example was one of our users who requested to go longer in order to be the only individual to complete all three levels. Based on the results, $87.5 \%$ of the users who were tested agreed that if more levels were provided in the game, they would use the VR game more. This would give them the option to go longer in the game without becoming bored since they can have a larger variety of scenery and game types (based on workout types) to choose from.

This paper presents a home-based system for integrating motion as it relates to spin machines or exercise bikes with a VR sensor and provide unique game mechanics that aim to improve both calorific output as well user experience. While there were some limitations in the use of the VR sensor, the design of the motion experience and the cost for building this setup at home, overall the findings have been positive and further research and development in this area is warranted. In our next phase we plan to test several commercial release versions of the VR sensors and increase variations in the game levels including ability for the users to set their own goals. We plan to compare these sensors with a larger group of targeted users and present the results of our research. Additionally we plan to also look at design mechanics that reduce the likelihood of players feeling dizzy during initial use of the system.

\section{References}

[1] Connolly, T. M., Boyle, E. A., MacArthur, E., Hainey, T., \& Boyle, J. M. (2012). A systematic literature review of empirical evidence on computer games and serious games. Computers \& Education, 59(2), 661-686.

[2] Deterding, S., Dixon, D., Khaled, R., \& Nacke, L. (2011, September). From game design elements to gamefulness: defining gamification. In Proceedings of the 15th international academic MindTrek conference: Envisioning future media environments (pp. 9-15). ACM.

[3] Prensky, M. (2001). Digital game-based learning.

[4] Bogost, I. (2005). The rhetoric of exergaming. Proceedings of the Digital Arts and Cultures (DAC).

[5] King, D., Greaves, F., Exeter, C., \& Darzi, A. (2013). 'Gamification': Influencing health behaviours with games. Journal of the Royal Society of Medicine, 106(3), 76-78.

[6] Lister, C., West, J. H., Cannon, B., Sax, T., \& Brodegard, D. (2014). Just a fad? Gamification in health and fitness apps. JMIR serious games, 2(2), e9.

[7] Pereira, P., Duarte, E., Rebelo, F., \& Noriega, P. (2014, June). A review of gamification for health-related contexts. In International Conference of Design, User Experience, and Usability (pp. 742-753). Springer International Publishing.

[8] Daley, A. J. (2009). Can exergaming contribute to improving physical activity levels and health outcomes in children?. Pediatrics, 124(2), 763-771.

[9] Siegel, S. R., Haddock, B. L., Dubois, A. M., \& Wilkin, L. D. (2009). Active video/arcade games (exergaming) and energy expenditure in college students. International journal of exercise science, 2(3), 165.

[10] Tanaka, K., Parker, J. R., Baradoy, G., Sheehan, D., Holash, J. R., \& Katz, L. (2012). A comparison of exergaming interfaces for use in rehabilitation programs and research. Loading..., 6(9). 
[11] Chen, J. (2007). Flow in games (and everything else). Communications of the ACM, 50(4), 31-34.

[12] Venkatesh, V. (2000). Determinants of perceived ease of use: Integrating control, intrinsic motivation, and emotion into the technology acceptance model. Information systems research, 11(4), 342-365.

[13] Staiano, A. E., Abraham, A. A., \& Calvert, S. L. (2012). Motivating effects of cooperative exergame play for overweight and obese adolescents. Journal of diabetes science and technology, 6(4), 812-819.

[14] Sun, H. (2012). Exergaming impact on physical activity and interest in elementary school children. Research quarterly for exercise and sport, 83(2), 212-220.

[15] Bailey, B. W., \& McInnis, K. (2011). Energy cost of exergaming: a comparison of the energy cost of 6 forms of exergaming. Archives of pediatrics \& adolescent medicine, 165(7), 597-602.

[16] Wilkinson, C., \& Hall, A. (2012). Designing and Using a High School Cardio Room. Strategies, 25(5), 12-15.

[17] Oculus, V. R. (2015). Oculus rift. Available from WWW: $<$ http://WwW.oculusvr.com/rift .
[18] VirZoom. (2015). VirZoom. Available from WWW:< http://www.theverge.com/2015/12/16/10234898/sonyplaystation-vr-virzoom-motion-controller-excercise.

[19] Amresh, A., \& Okita, A. (2010). Unreal Game Development. CRC Press.

[20] LaValle, S. M., Yershova, A., Katsev, M., \& Antonov, M. (2014, May). Head tracking for the Oculus Rift. In Robotics and Automation (ICRA), 2014 IEEE International Conference on (pp. 187-194). IEEE.

[21] Bolton, J., Lambert, M., Lirette, D., \& Unsworth, B. (2014, April). PaperDude: a virtual reality cycling exergame. In CHI'14 Extended Abstracts on Human Factors in Computing Systems (pp. 475-478). ACM.

[22] Desai, P. R., Desai, P. N., Ajmera, K. D., \& Mehta, K. (2014). A review paper on oculus rift-A virtual reality headset. arXiv preprint arXiv:1408.1173.

[23] Warren, J. D., Adams, J., \& Molle, H. (2011). Arduino for robotics (pp. 51-82). Apress. 